\title{
Editorial
}

\section{Multifaceted Social Work in context}

By

Kjersti Ørvig

Associate Professor PhD

University of Stavanger

Email: kjersti.orvig@uis.no

Memory J. Tembo,

Research fellow

University of Stavanger

Email: memory.j.tembo@uis.no

\section{(c) (i) (?)}

This work is licensed under a Creative Commons Attribution-ShareAlike 4.0 International License. 
We are glad to invite our readers to this year's second issue. All the contributions tackle the challenges facing social work in the different countries presented in this issue.

In the wake of public sector reforms, the work environment of professionals is changing. This is the starting point of the first article from Norway by Jorunn Theresia Jessen. The article: Public governance-constraints and challenges for social work practice investigates the extent of managerial and administrative regulation in the Norwegian social services, questioning the asserted negative impacts on professional autonomy in social work practice. The data is collected from a survey she conducted with practitioners and managers in 125 local agencies, and the findings of her study reflect a dichotomy that is documented in earlier studies. On the one hand, this dichotomy reveals the effectivity and outputs defined in measurable and quantifiable performance indicators, while on the other the new management models allows for more autonomy and flexibility in the use of allocated resources and in the choice of means and measures. Furthermore, the findings indicate that professional fellowship and collegial authority play a role in guiding and regulating social work practice.

The second article is about the perspectives of Canadian parents of adopted children from China by Fang Bian, Dongyan Blachford and Douglas Durst. The authors of this article uncover the beliefs and attitudes of the adopting parents about how to best promote a healthy identity development that includes their Chinese background. By using in-depth interviews of 19 parents of 14 adopted children, they explore their beliefs about incorporating Chinese heritage in the development of their girls. The data reveals four different types of identity that parents form for their children. Firstly, some parents feel that it is best to emphasize Canadian culture; secondly, parents feel that it is best to let the children choose their "identity"; thirdly, parents become proactive by introducing Chinese heritage and culture to their children at an early age. Fourthly, and more interesting, is the formation of the "color purple". In this scenario, parents feel that their children have created a new identity of the color purple. This means a hybrid and new culture that combines both their Chinese background with a mainstream Canadian lifestyle.

Many organizations in Malawi are working towards HIV awareness among the population to prevent the transmission of HIV. In this article, Inger Sofie van Pelt and 
Anne Ryen explore the complexity of health behavior from a self-efficacy perspective, and show the naivety in assuming knowledge as the main guide to a better protection against HIV. The authors explored how female university students in Malawi perceive their own self-efficacy in protecting themselves against HIV in Malawi. The study is based on data from Chancellor College in Zomba Malawi, with 17 semi-structured interviews and three group interviews. The article is rich with extracts from the interviews, and reveals three analytical categories: Relational communication, social expectations and practical protection. The article discusses the findings of this study through an expansion of these three categories based on the extracts from the data material. The authors point to the potential of a closer collaboration between social work and public health in issues of both a social and a health nature, as in the case of HIV protection.

Are we right to blame it all on colonialism? The subject of history and gender in schools in a Malawian context. This is another article from Malawi by Alinane Priscilla Kamlongera Katenga-Kaunda. In this article, Alinane explores the influence of colonialism on the history/social and environmental sciences in primary school subjects. The study emerges largely from a text analysis of selected books, combined with a count of the number of women compared to men, as well as a count of the feminine pronouns against masculine pronouns in literature. The article discusses the dominance of the usage of male pronouns in most books. She explains how the syllabuses show that women compared to men have been portrayed as people who did not play an integral part in the formulation of history. The findings of her study reveal how different agents have influenced how the curriculum has been written in regard to content and gender. She questions the tendency to blame the dominance of male in history on colonialism by looking at other factors that might have caused the dominance of "his" story in history.

The last article in this issue, written by Angela Pena and Rosa Maria Voghon, focuses on social work with families in Cuba and the process of the construction of concepts in social work. They look at how social workers in a Cuban context define family, and how this definition affects their practice when working with families. Concerning the definition, their findings show that the social workers in a Cuban context define family as a group of cohabitation and affinity, which is more important than blood relationship 
Journal of Comparative Social Work 2015/2

as dimensions for family definitions. The data is based on a qualitative research design collected through two group interviews with social workers, with 11 participants in the two groups. Regarding family as a field of practice, they all share the criteria that practice is necessary to develop a contextual analysis of each situation, which goes from macro-contextual aspects to the micro-reality of family. 